zinkpräparaten ist außerordentlich bequem und zuverlässig. Die Chlorzinkpräparate geben - wie der Praktiker sich ausdrückt - einen guten Fluß, das Lot haftet leicht und sicher auf der Lötstelle, so daß bei einiger Geschicklichkeit ein Nacharbeiten (Putzen) der Lotnähte vermieden werden kann. Auch wenn die zu verbindenden Oberflächen mit starken Oxydschichten bedeckt sind, gelingt die Lötung bei einiger Geschicklichkeit zuverlässig und sicher. Um möglichen Nachwirkungen zu begegnen, ist es üblich, die Verbindungsstellen nach dem Löten mit Zinkchlorid abzuwaschen.

In der bereits angezogenen Kontroverse wurde versucht, Chorzinkpräparate als ebenso gefährlich hinzustellen, als man Chlorammonium als ungefährlich bezeichnen wollte.

Abgesehen davon, daß $\mathbf{S a} \mathbf{m}$ te $\mathbf{r}$ auch zu diesem Punkte bereits hinreichende Aufklärung gegeben hat, haben auch die praktischen Versuche hier das Gegenteil ergeben. Mit Chlorzinkpräparaten abgelötete Kabelverbindungen wurden in gleicher Weise aufbewahrt und nach Ablauf derselben Zeitabschnitte geöffnet, wie dies mit den Salmiakproben geschehen war. Der Unterschied war aber ein ganz erheblicher. Auch hier war das im Überflu $\beta$ vorhandene Flußmittel in das Innere der Drahtbunde gelıufen und nicht entfernt worden. Die Seelendrähte zeigten einen wachsartigen Ủberzug von schwach dunkelgrïner Färbung, der sich trocken anfühlte. An Stellen, an denen durch Überhitzen die in der verwendeten Chlorzinkseife enthaitenen anderen Stoffe verbrannt worden waren, zeigte sich ein pechartiger Ùberzug, der sich auch nach längerem Liegen nicht veränderte.

Die Verbindungen, die längere Zeit hindurch einer erheblichen Strombelastung ausgesetzt worden waren, zeigten keine nachweisbare Widerstandsänderung.

Auch die Behauptung, daß die sich zufolge der hygroskopischen Eigenschaften des Chlorzinks hydrolytisch abspaltende Salzsäure nachteilige Wirkungen ergeben müsse, wurde experimentell geprüft. Es wurden mit dünnen Kupferdrähten von unter $0,1 \mathrm{~mm}$ Durchmesser Lötstellen hergestellt, diese mit dem Chlorzinkpräparat dick bestrichen und unter Strombelastung in der in meiner Arbeit (Elektrotechn. Z. 1907, 875) erläuterten Thom s o n schen Schaltung an die Meßbrücke gelegt. Nach einigen Tagen wurden die aufgeschmierten Flußmittelreste feucht, trockneten aber bald wieder ein, und es verblieb auf der Lötstelle die schon beschriebene, wachsartige Masse, die sich nicht weiter veränderte. Die Drähte waren frei der umgebenden Atmosphäre, die stets einen nicht unerheblichen Feuchtigkeitsgehalt zeigte, ausgesetzt; die längere Zeit hindurch geführten Beobachtungen ließen eine Schädigung nicht erkennen. Es kann somit auf Grund von mehrjährigen erschöpfenden Versuchen behauptet werden, daß Chlorzinkpräparate in der Löttechnik gegenüber den Salmiakpräparaten in jeder Hinsicht den Vorzug verdienen. Auch kann kein Zweifel darüber bestehen, daß es lediglich einer Selbsttäuschung gleichkommt, falls man ans der Tatsache, daß3 sich Salmiakpräparate säurefrei herstellen lassen, die Unschädlichkeit der Salmiaklösungen abzuleiten sucht, zumal da die starke Angriffsfähigkeit der Salmiaklösungen Metallen gegenüber ja nicht der verháitnismäßig harmlosen Salzsäure, sondern den erwähnten anderen Ursachen zuzuschreiben ist.

Welchen Ursachen die außerordentlich günstige praktische Wirkung der Chlorzinkpräparate beim Lötprozeß $\mathrm{zu}$ verdanken ist, wäre allerdings noch aufzuklären. Das dürfte aber eine Aufgabe des chemikers sein.

[A. 123.$]$

\section{Apparate zur Schwefelbestimmung in Eisen und Stahl.}

Von Georg Predss in Gelsenkirchen-Schalke.

(Eingeg. 21.18. 1909.)

Es ist stets das Bestreben eines jeden Analytikers bei Ausführung von Schwefelbestimmungen gewesen, der Sicherheit halber ohne jede Gummiverbindung arbeiten zu können. Einen solchen von mir konstruierten Apparat, welcher auch den Vorzug besitzt, wenig Raum einzunehmen, will ich im Folgenden beschreiben.

\section{Apparat Nr. I.}

Der Kolben, welcher zur Aufnahme des Materials dient, ist mit einem Ansatzrohr zur Einführung der Salzsäure und nachherigem Durchleiten von Kohlensäure versehen. Dieses ist ja auch bekannt.

Auf dem Kolben befindet sich der Kühler eingeschliffen und in diesem das Absorptionsgefäß. Das im Innern des Kühlers angeschmolzene Schlangenrohr leitet die entwickelten Gase unter vollständiger Kondensation der Salzsäuredämpfe durch das Ventilrohr zum Absorptionsgefäß. Oben in dem Ventilrohr ist ein eingeschliffenes Rückschlagventil angebracht, wodurch ein Zurücksteigen der Flüssigkeit aus dem AbsorptionsgefäßB vollständig verhindert wird.

Die Anwendung des Apparates ist folgende:

Nachdem der Kolben mit $10 \mathrm{~g}$ Stahl oder $5 \mathrm{~g}$ Roheisen versehen ist, wird derselbe mit dem Kühler verschlossen, mit Wasser abgedichtet und das Absorptionsgefäß mit $40 \mathrm{ccm}$ Cadmiumlösung gefüllt. Durch das seitlich am Kolben angebrachte Säurezuführungsrohr worden nun 150 oder $75 \mathrm{ccm}$ verd. Salzsäure vom spez. Gew. 1,12 eingelassen und das Material unter schwacher Erhitzung zum Lösen gebracht. Man verjagt den Rest des Gases durch einen Kohlensäurestrom von kurzer Dauer, und die Operation ist beendet. Die Úberführung des Schwefelcadmiums aus dem Absorptionsgefäß in ein Becherglas geschieht dadurch, daß man den Kühler aus dem Kolben hebt. während der Kolben eingeklemmt auf dem Drahtnetz stehen bleibt. Nach dreinaligem Umspülen mit Wasser ist das Absorptionsgefäß vollständig von jeder anhaftenden Spur Schwefelcadmiums befreit. Auch hierbei tut der kleine Schwimmer seine Schuldigkeit als Abschlußventil, indem er beim Entleeren nichts in die Schlange treten läßt. Das in das Becherglas überführte Schwefelcadmium wird in Schwefelkupfer umgesetzt, filtriert und nach der gewichtsanalytischen Methode bestimmt.

Dieser Apparat genügt allen für die Betriebsanalyse gestellten Anforderungen. Da jedoch bei 
Anwendung von konz. Salzsäure vom spez. Gew. 1,19 trotz der guten Kühlung nicht alle Salzsäuredämpfe kondensiert werden, konstruierte ich den Apparat Nr. 2.

\section{Apparat Nr. 2 .}

Dieser Apparat ist genau wie $\mathrm{Nr}$. 1, nur hat derselbe unterhalb des Kühlers noch eine Wasservorlage, als Waschflasche dienend. Die Lösung des Materials in diesem Apparat kann mit konz. Salzsäure vom spez. Gew. 1,19 vorgenommen werden, da bei der Operation die nit $50 \mathrm{ccm}$ beschickte Waschflasche die Hauptmenge der übergehenden Salzsäure zurückhält. Auch hat man nicht zu befürchten, daß Schwefelwasserstoff darin absorbiert bleibt, da die Flüssigkeit sich fast bis zum Sieden erwärmt.

Die Fiillung dieser Wasservorlage geschieht am besten mit einer Spritzflasche, durch die unter dem eingeschliffenen Teil angebrachte Öffnung, von der

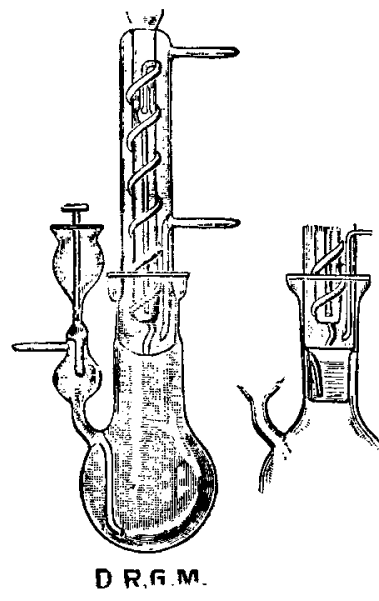

Apparat 1. Apparat 2. ein Röhrchen bis fast auf den Boden der Vorlage geht. Nach vollendeter Operation entleert sich diese Vorlage ganz von selbst und fließt in den Kolben zurück, während das Rückschlagventil ein Zuriicksteigen des Schwefelcadmiums aus dem Aborptionsgefäß verhindert.

Die Apparate wurden von mir auf das genaueste ausprobiert mit einem $\mathrm{Ma}$ terial von Normalstählen, derenSchwefelgehalt bekannt war. Bei allen Versuchen waren die Resultate mit den alten übereinstimmend; nur bei Anwendung von konz. Salzsäure im Apparat Nr. 2 wurden die Werte, wie bekannt, ein klein wenig höher ${ }^{1}$ ).

Erwähnt sei noch, daß die Wasserkühlung bis in den eingeschliffenen Teil des Apparates hinuntergeht, und somit ein Festsetzen des Schliffes ausgeschlossen ist. Um acht dieser Apparate mit gleichmäßigem Kohlensäurestrom auf einmal bedienen zu können, wurde der $\mathrm{K}$ i p p sche Gasentwicklungsapparat von mir abgeändert. Es ist ein großer Apparat von 51 Inhalt, an jeder Seite mit einem Tubus und je 4 Hähnen zur Stromentnahme versehen.

Forstehend beschriebene Apparate sind unter D. R. G. M. Nr. 384317 vor kurzem gesetzlich geschützt und werden von der Firma $S$ t $r$ ö h le i n \& Co. in Düsseldorf hergestellt.

[A. 139.]

1) Siehe auch Stahl und Eisen, Heft $\mathrm{Nr} .8$ vom 19./2. 1908. Schwefelbestimmung usw.

\section{Automors, ein neues Desinfektionsmittel. Von Dr. P. Flemmina.}

Wohl jedem Fachgenossen sind im Laufe der letzten Zeit die in der Fach- und Tagespresse erschienenen Inserate über Automors in die Augen gesprungen, und mancher von uns hat sich gewiß schon die Frage vorgelegt: Was ist Automors? und wie unterscheidet es sich von den bisher bekannten Desinfektionsmitteln?

Unter diesem Umständen dürfte die Veröffentlichung einiger vom Verf. gesammelter Daten für die die Medizinisch-Pharmazeutische Literatur verfolgenden Kollegen von Interesse sein:

In einer an die Redaktion des ,Drogenhändler" gerichteten Zuschrift äußert sich die Automorsfabrik über die Zusammensetzung ihres Präparates folgendermaßen: „Es handelt sich um gewisse Verbindungen sehr geringer Mengen Kresole mit Säuren." Ferner versendet die Fabrik eine Brosehüre mit der Aufschrift "Was ist Automors" und einem großen Fragezeichen. Eine Antwort gibt der Text der Broschüre aber nicht. In der vorerwähnten Zuschrift ist von einer Patentschrift die Rede, aus welcher eventuell Näheres über das Wesen des neuen Mittels zu erfahren wäre, doch hat die betreffende Redaktion trotz eifrigen Suchens eine solche Patentschrift nicht ermitteln können, vielmehr eine Warenzeicheneintragung, aus der sich naturgemäß für den Gegenstand nichts ergibt. So mußte also auf die Analyse zurückgegriffen werden, um in Erfahrung zu bringen, was in dem neuen Mittel eigentlich vorliegt. Aus einer von Dr. Nördlinger veranlaßten Untersuchung ergibt sich, daB 2 zu verschiedenen Zeiten gekaufte Proben des Automors wechselnde Mengen von Phenol, Phenolschwefelsäure und freier Schwefelsäure enthielten. Eine von Dr. M üll er, Hamburg vorgenommene Untersuchung ergab $1 \%$ Kohlenwasserstoffe, 2,5\% Phenol, 0,85\% Amine, ca. $17,5 \%$ freie Schwefelsäure, ebensoviel Kresolschwefelsäure sowie etwas schweflige Säure.

Eine von Dr. H e rold, Flörsheim, vorgenommene ausführliche Untersuchung ergab folgendes..Resultat: Automors ist eine leicht bewegliche schwarzbraune Flüssigkeit, die schwach nach Schwefeldioxyd and Teeröl riecht. Spezifisches Gewicht bei $15^{\circ}$ 1,202. Freie Schwefelsäure 15,43\%, Sulfosäuren a!s Schwefelsäure berechnet 11,43, schweflige Säure in Spuren, Asche 0,33, Harz 0,16, organische nicht sulfurierte Körper meist Kohlenwasserstoffe neben Phenol 1,80, Wasser ca. 50.

Es ergibt sich also zunächst einmal, daß Automors überhaupt keine einheitliche Zusammensetzung besitzt, und ferner, daß es anscheinend durch Mischen von Rohkresol mit Schwefelsäure in wechselnden Mengen unter nachfolgendem Frhitzen gewonnen werden dürfte, und zwar scheint ein sehr teerhaltiges Kresol Verwendung zu finden, wenigstens deutet darauf der klebrige Úberzug, der sich aus Automorslösungen auf alle damit in Berührung kommenden Gegenstände beim Stehen ablagert, sowie die nicht klare Löslichkeit des Produktes in Wasser, während selbst ein 90\%iges Rohkresol mit der entsprechenden Menge Schwefelsäure vermischt, noch ein vollkommen klar lös- 